\title{
Measurement of PD in Operating Motors Fed by Voltage Source PWM Drives
}

\author{
H.G. Sedding, I. Culbert, S.R. Campbell \\ Qualitrol-Iris Power \\ Mississauga, Ontario, Canada
}

\begin{abstract}
On-line partial discharge (PD) measurement is now widely applied to motors rated $6 \mathrm{kV}$ and above that are fed from the 50/60 $\mathrm{Hz}$ power system. However, many motors are being powered from invertors which facilitate variable speed motors to improve process efficiencies. One of the most common drives now being used is of the voltage source, pulse width modulation (VS-PWM) type. Such drives generate high voltage spikes in the $\mathrm{kV}$ range with risetimes in the submicrosecond range. These high voltage spikes are a form of severe electrical interference which render the detection of PD (with magnitudes $1000 \mathrm{x}$ smaller) difficult, due to the overlapping frequency content in PD and the spikes. Thus PD detection on VS-PWM has been a challenge, in spite of the serious insulation aging that such drives may cause. This paper will outline the challenges in measuring the stator winding $\mathrm{PD}$ in motors fed by VS-PWM drives and describe a measurement system that is often effective. Results from measurements obtained using this system will be presented.
\end{abstract}

\section{Introduction}

The application of voltage source, pulse width modulated (VS-PWM) drives, on medium and high voltage motors ( $2.4 \mathrm{kV}$ to $13.8 \mathrm{kV}$ ) is increasing. Other drive topologies, such as load commutated and current source pulse width modulated schemes are also available, however, due to cost considerations, VSPWM drives are becoming more common. Unfortunately, these drives have resulted in stator winding insulation problems [1 - 7]. This observation is due to such inverters producing bipolar, short risetime voltage pulses with a high repetition rate. In order to ameliorate this effect in medium and high voltage stator windings, multi-level topologies for converters have been implemented. Thus, as the rated voltage increases so does the number of levels. As discussed below, the voltage impulses from the drive can lead to rapid stator insulation system aging [4 - 7]. In most cases, as the aging progresses the PD activity increases. Thus, just as for conventional power frequency motors, there is a desire for reliable on-line PD detection methods for these medium and high voltage motors. Unfortunately, conventional electrical PD detectors tend to be overwhelmed by the 1000-3000 V impulses created by multi-stage VS-PWM drives. The risetime of the voltage impulses from the multi-stage VS-PWM drives tend to be 500 ns or longer. This paper describes the technical challenges in implementing on-line PD detection on motors fed by VS-PWM drives and provides some examples of application on operating IFD motors.

\section{Consequences of Inverter Fed Drives on Form Wound Stators}

Experience over the past decade indicates that the voltage impulses from the VS-PWM inverters can age each component of the stator insulation system differently. These adverse effects were described in detail elsewhere [6], however, briefly,

\subsection{Turn Insulation Deterioration}

If there are voids associated with the turn insulation and if the interturn voltage is of sufficient magnitude, then PD can occur. This mechanism is less of a concern in form-wound stators for VS-PWM applications if mica paper turn insulation is used, which is very resistant to PD.

\subsection{Ground and Phase Insulation}

The short-risetime switching voltage and the associated transmission line reflection effects may lead to voltage doubling, which in turn, results in the peak-voltage to rms-voltage ratio being higher than the 1.4 ratio that is present with sinusoidal voltage $[4,6]$. The higher peak voltage will increase the repetition and magnitude of groundwall insulation PD and thus accelerate aging. Further, the number of PD pulses may also be higher than for a conventional $50 / 60 \mathrm{~Hz}$ motor since the switching frequency is $1000 \mathrm{~Hz}$ or more and each resulting impulse may create PD in a void.

\subsection{Stress Relief Coatings}

Since inverters generate high-frequency voltages, the capacitive currents through the ground insulation are relatively high compared to power frequency. The highfrequency capacitive currents will result in high local $\mathrm{I}^{2} \mathrm{R}$ losses in the coatings compared to power frequency windings [4 - 7]. The result is that the stress control layers in VS-PWM inverter fed motors are likely to deteriorate much faster than the layers in $50 / 60 \mathrm{~Hz}$ windings due to the resulting high temperature. As the stress relief coatings deteriorate, they will create PD activity. 


\section{Measuring PD On-Line in IFD Motors}

Off-line and on-line PD testing of conventional motors has been commonly practiced using a range of measurement technologies. The various methods employed are largely standardized [8 - 10]. However, the VS-PWM type of drive produces significant shortrisetime voltage impulses. Such drives use IGBT (or similar) switching transistors, which switch DC voltages of $1000-1500 \mathrm{~V}$. With worst case transmission line voltage reflections of 2 times, impulse voltages may be up to $3000 \mathrm{~V}$. Due to the several levels of medium and high voltage inverters, the risetime of these impulses has been measured to be about 500 ns or more. Using a Fourier transform, a 500 ns risetime impulse will produce frequencies up to about $600 \mathrm{kHz}$. Future VSPWM drives are likely to have shorter switching times and higher switching voltages, making the situation worse.

These steep-fronted pulses are problematic because they have frequency components that are within the range of those associated with PD while having magnitudes 1000 times (or $60 \mathrm{~dB}$ ) higher than that of PD signals. In power frequency applications, the $\mathrm{mV} \mathrm{PD}$ pulses are most commonly separated from the high voltage 50/60 $\mathrm{Hz}$ using a single-pole high-pass filter. With a PD detector operating in the VHF range, the high voltage $50 / 60 \mathrm{~Hz}$ is reduced to $<10 \mathrm{mV}$, while the magnitude of the $\mathrm{PD}$ (which in air at $100 \mathrm{kPa}$ has a risetime of about 3 ns) is largely unaffected. In contrast, a 500 ns risetime, $3000 \mathrm{~V}$ impulse may be reduced to only about 10 volts by a single pole high pass filter with a lower cut-off frequency of $40 \mathrm{MHz}$. Detectors operating in the $\mathrm{HF}$ (3-30 MHz) and $\mathrm{LF}(<3 \mathrm{MHz})$ range will have even higher residuals from the voltage impulses. Thus, additional electronic or passive filters or other means are needed to further suppress the impulse voltage another $60 \mathrm{~dB}$ or so in order to reduce the residual to less than the typical PD levels (using a VHF measurement method, significant PD has a magnitude of several hundred $\mathrm{mV}[7])$.

Methods to circumvent this problem and enable PD detection on low voltage IFD motors are described elsewhere [11]. However, as described in [3], the methods that have proved successful for low voltage random-wound motors may not be effective for medium and high voltage motors.

Another practical issue with PD detection in medium and high voltage VS-PWM motors is providing a voltage proportional to the fundamental frequency coming from the inverter, since it is traditional to display PD with respect to the AC sinewave. For conventional motors, the power frequency sinewave can be extracted from the PD coupler, and phase corrected for the 90 degree phase shift. Experience has shown that it is difficult to reliably synchronize to the fundamental voltage with the high magnitude voltage impulses superimposed on the fundamental AC voltage. An extremely narrow band filter would not work since the frequency of the fundamental voltage varies over a wide range (for example 10-60 Hz). An example of a typical voltage reference signal obtained from a capacitive divider is shown in Figure 1 . The characteristics of this voltage output are such that it is of little use as a phase reference. This problem can be solved using a capacitive voltage divider and by introducing additional capacitance to the filtering to lower the cut off frequency, Figure 2.

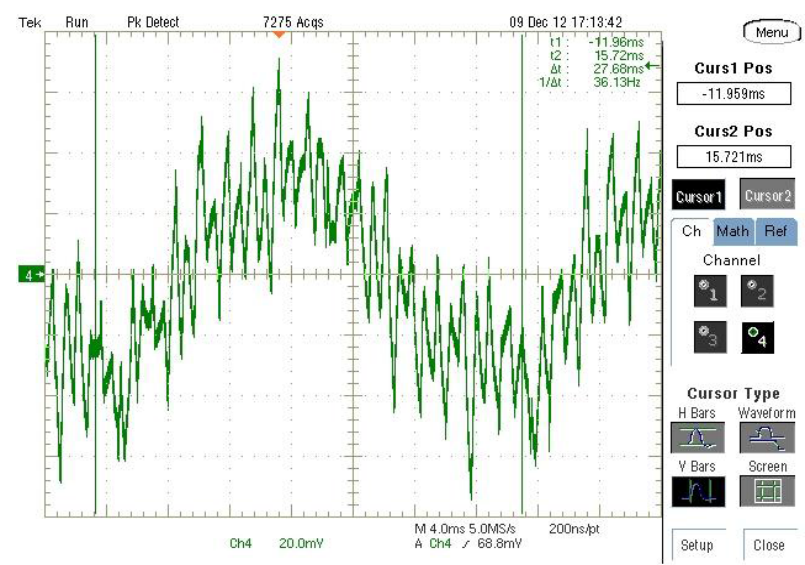

Fig. 1 - Example of voltage reference signal obtained from capacitive divider at motor terminals.

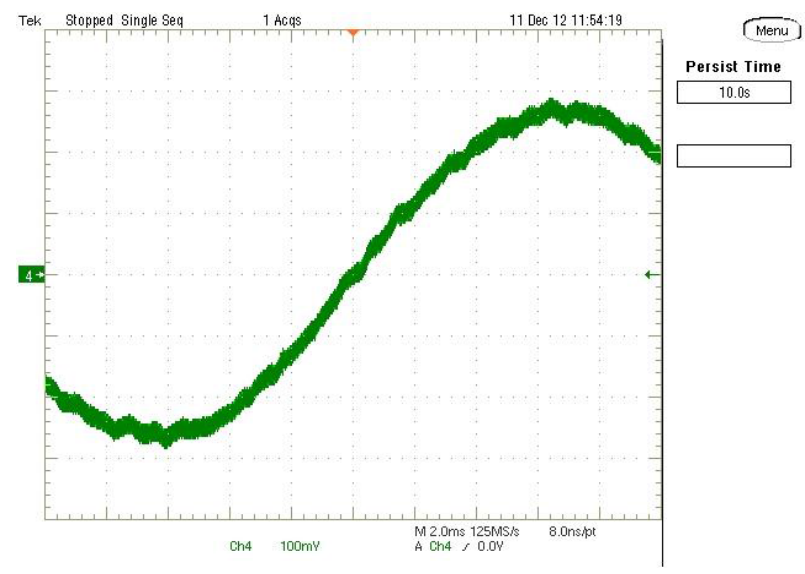

Fig. 2 - Signal from the capacitive divider with changes to the filter circuit.

\section{An On-Line PD System for Medium and High Voltage Inverter Drives}

The system used to derive the measurements discussed in this paper was based on a conventional VHF on-line PD detector rather than on the UHF system developed for low voltage stators. The $80 \mathrm{pF}, 16 \mathrm{kV}$ capacitors use a mica splitting dielectric which yield impulse flashover voltages $>100 \mathrm{kV}$ and meet all the requirements of IEC 60034-27-2 and IEEE 1434 for rotating machine on-line PD sensors (Figure 3). Figure 4 , illustrates the output of these PD sensors without filtering (brown trace), with a $500 \mathrm{kHz}$ filter (purple trace) and the filtered voltage reference signal (green 
trace). In this case, the voltage spikes from the inverter are over $20 \mathrm{~V}$.

As mentioned above, a practical challenge in implementing reliable on-line PD monitoring of VSPWM drive motors is the derivation of a voltage reference signal to enable phase resolved measurements. Most modern PD instruments require such a signal in order to be functional. In several prototype installations, a capacitive voltage divider was optimized for the frequency range $150 \mathrm{~Hz}$ and below and placed at the inverter output. However, often the inverter is located up to several hundred metres from the motor which causes not only installation difficulties but also attenuation of the detected high frequency signals that may interfere with reliable measurements.

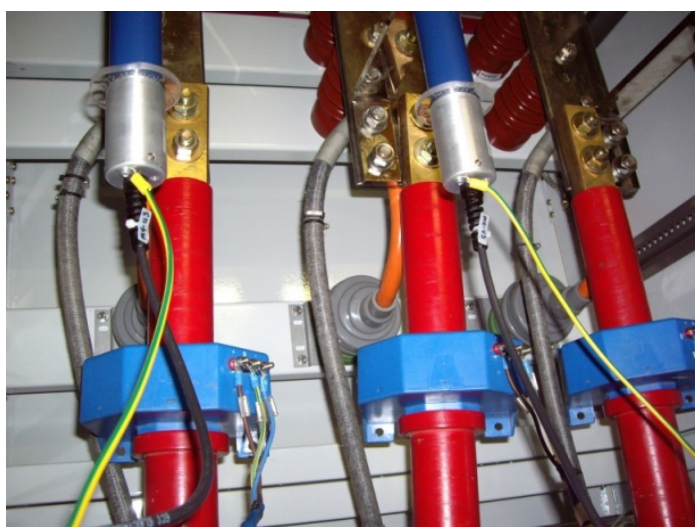

Fig. 3 - Installation of the $80 \mathrm{pF}$ capacitive sensors on the motor terminals.

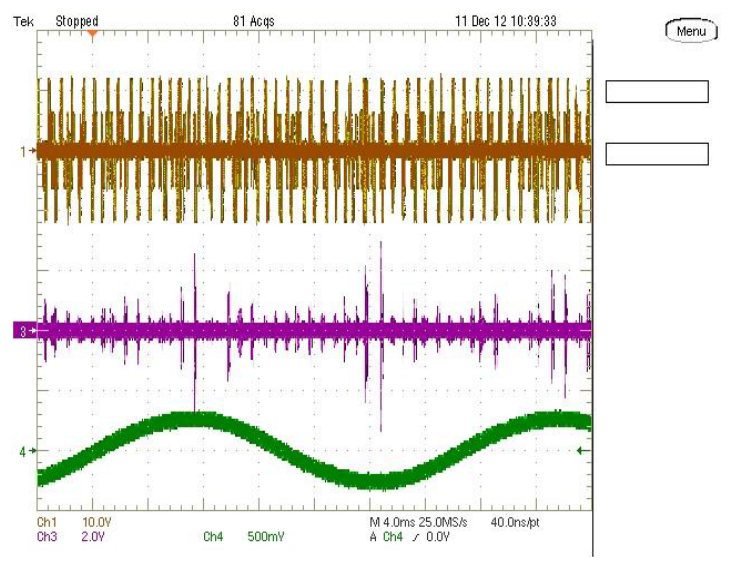

Fig. 4 - Oscilloscope traces from a $12.5 \mathrm{MW}, 3 \mathrm{kV}$ motor at the output of the PD sensors and capacitive dividers measured across a $50 \mathrm{ohm}$ resistor. The top two traces represent the PD and noise signals, unfiltered and filtered respectively. The bottom trace is the AC voltage measured on phase A ( $5 \mathrm{~V}$ per division) from a capacitive voltage divider at the drive output. The horizontal scale is $1 \mathrm{~ms}$ per division.

\section{Case Studies}

A VHF PD detection system, as described above, was deployed on a number of $12.5 \mathrm{MW}, 3 \mathrm{kV}$ compressor motors.
The waveform of the impulse voltage measured at the stator using a high voltage divider is shown above in Figure 4 (lower trace). Figure 4 also shows the unfiltered and filtered signals from one of the three PD sensors.

Figure 5 provides an example of the output from three phases of one of the motors. Examination of Figure 5 indicates that there is no PD of any significance present. The pulses evident on the PRPD plots are the residual components of the high voltage impulses from the drive system. Note that there is no correlation between the pulses and the phase position. This motor stator winding is new so the lack of PD on a relatively low voltage machine is perhaps not surprising. The presence of noise pulses, despite significant efforts to reduce electrical interference from the drive, is a further indication that performing PD measurements on operating IFD motors is non-trivial.

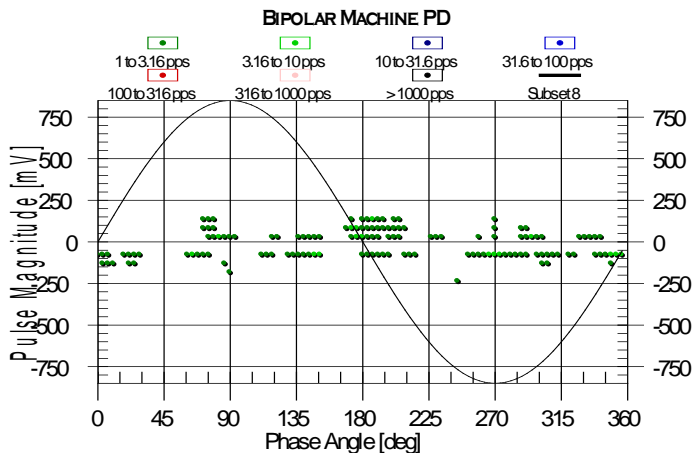

Fig. 5(a)

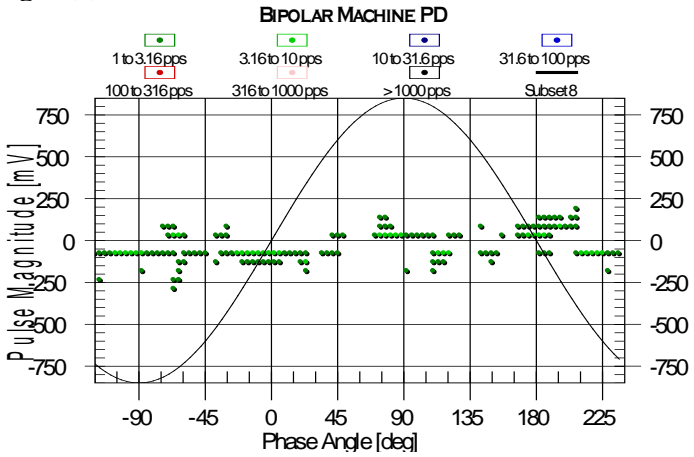

Fig. 5(b)

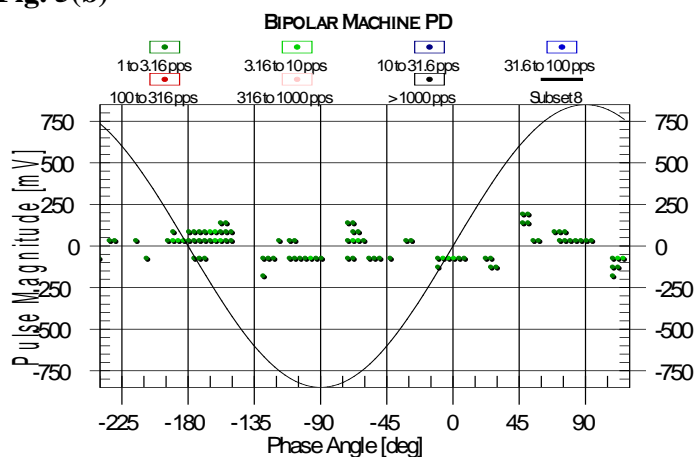

Fig. 5(c)

Fig. 5 - Signals detected from the three phases of a $12.5 \mathrm{MW}$, $3 \mathrm{kV}$ motor fed by a VS-PWM converter. 
On-line PD testing was performed on several $45 \mathrm{MW}$, $7.2 \mathrm{kV}$ compressor motors fed by VS-PWM drives. Figure 6 illustrates the phase-resolved PD signal measured by the modified VHF detector on three of these motors during operation. Examination of the PD plots in Figure 6 shows that, in these cases, they exhibit similar characteristics data derived from measurements in power frequency applications. Further inspection of the data contained in Figure 6 shows that the peak PD magnitudes recorded on these motors ranges from 500 to $1200 \mathrm{mV}$. Comparison of these values with a database containing over 400,000 on-line PD test results [12] shows that when the peak PD exceeds $350 \mathrm{mV}$ (for this voltage class of motor), further off-line tests and inspections are warranted. Consequently, these stator windings appear to have significant PD activity.

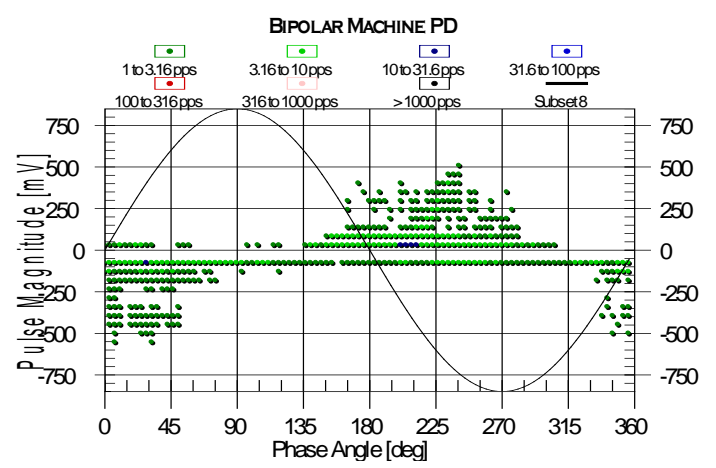

Fig. 6(a)

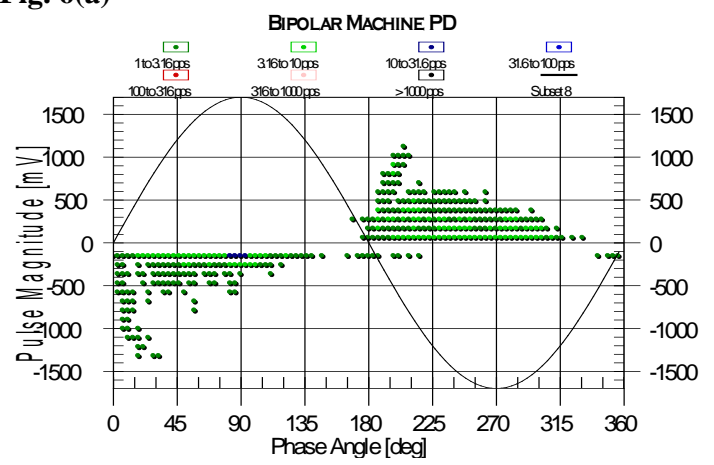

Fig. 6(b)

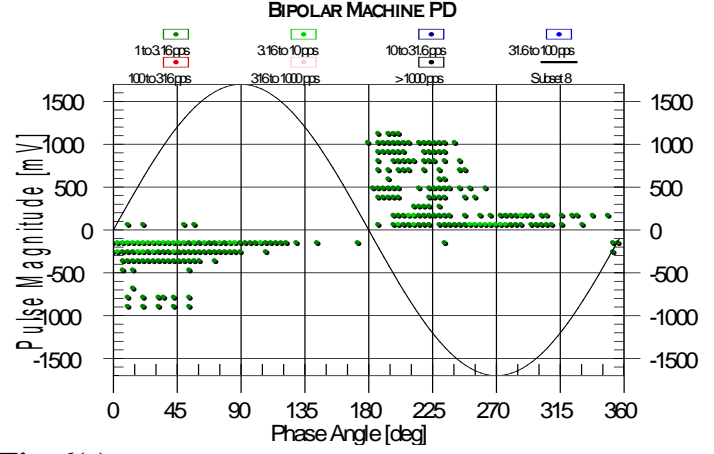

Fig. 6(c)

Fig. 6 - AC phase resolved PD patterns from the modified VHF detector on three different $7.2 \mathrm{kV}, 45 \mathrm{MW}$ motors fed by a VS-PWM converter. The color displays the PD pulse repetition rate.

\section{Conclusion}

1. On-line motor PD measurement on newer VSPWM drives can be achieved, however, significant effort is required to suppress the high voltage impulses associated with the drive electronics. Further, derivation of a reliable phase reference signal is also non-trivial.

2. Taking into account these challenges, a conventional on-line VHF PD system was modified to measure the stator winding PD on a number of 3 $\mathrm{kV}$ and $7.2 \mathrm{kV}$ stators connected to VS-PWM drives.

3. Results obtained to date are promising; however, further installations and data are required in order to better understand the PD pulse patterns obtained from these drives.

\section{References}

[1] E. Persson, "Transient Effects in Applications of PWM Inverters to Induction Motors," IEEE Trans. Industry Applications, p 1095, Sept. 1992.

[2] IEC 60034-18-41, "Rotating electrical machines Part 18-41: Qualification and type tests for Type I electrical insulation systems used in rotating electrical machines fed from voltage converters”.

[3] G. C. Stone, I. Culbert and S. R. Campbell, "Progress in On-Line Measurement of PD in Motors Fed by Voltage Source PWM Drives," in Proceedings of IEEE Electrical Insulation Conference, Philadelphia, June 2014, pp 172-175.

[4] IEC 60034-18-42, "Rotating electrical machines: Qualification and acceptance tests for partial discharge resistant electrical insulation systems (Type II) used in rotating electrical machines fed from voltage converters".

[5] E. Sharifi, S. Jayaram, E. Cherney, “Analysis of Thermal Stresses in Medium Voltage Motor Coils Under Repetitive Fast and High Frequency Pulses", IEEE Trans DEI, 2010, pp 1378-1384.

[6] M. Stranges, G.C. Stone, D. Bogh, "Voltage Endurance Testing of Stator Insulation Systems for Inverter Fed Machines", IEEE Industry Applications Magazine, Nov 2009, pp 12-18.

[7] G. C. Stone et al, "Electrical Insulation for Rotating Machines - Second Edition”, Wiley - IEEE Press, 2014.

[8] IEC 60034-27, “Off-line partial discharge measurements on the stator winding insulation of rotating electrical machines".

[9] IEC 60034-27-2, “On-line partial discharge measurements on the stator winding insulation of rotating electrical machines".

[10] IEEE 1434, "Guide to the measurement of partial discharges in rotating machinery".

[11] IEC 61934, "Electrical insulating materials and systems - Electrical measurement of partial discharges (PD) under short rise time and repetitive voltage impulses".

[12] V. Warren, "Partial discharge testing: a progress report”, Proceedings of 2014 Conference on Online Monitoring of Electrical Assets, Austin, TX, December 2014. 